\title{
La transposition didactique du spectacle professionnel comme objet de savoir en classe d'arts : proposition d'un modèle d'analyse intégratif
}

\author{
Anne Nadeau ${ }^{1}$, Caroline Raymond ${ }^{1}$ et Martin Lépine ${ }^{2}$ \\ ${ }^{1}$ Université du Québec à Montréal, Québec, Canada \\ ${ }^{2}$ Université de Sherbrooke, Québec, Canada
}

\section{Pour citer cet article :}

Nadeau, A., Raymond, C. et Lépine, M. (2021). La transposition didactique du spectacle professionnel comme objet de savoir en classe d'arts : proposition d'un modèle d'analyse intégratif. Didactique, 2(2), 41-62. https://doi.org/10.37571/2021.0203.

Résumé : Dans le cadre scolaire, les disciplines associées au domaine des arts se distinguent par leur nature praxique. Dans ce contexte, les pratiques artistiques des personnes enseignantes spécialistes des arts, que ce soit comme créatrices, interprètes ou spectatrices, influencent leur processus de transposition didactique. Cet article présente comment un spectacle professionnel apprécié par les spécialistes des arts est considéré comme un objet de savoir et comment il peut se transposer en classe. En croisant deux modèles théoriques, soit la triple dimension de l'enseignement des arts (Raymond, 2014, 2018) et le rapport au spectacle (entre autres inspiré des travaux d'Émery-Bruneau, 2010, 2014), nous proposons un modèle d'analyse intégratif de la transposition didactique, en classe, du spectacle professionnel en fonction du rapport qu'entretiennent les personnes enseignantes avec cet objet de savoir et des dimensions artistique, esthétique et culturelle propres au domaine des arts.

Mots-clés : enseignement des arts, transposition didactique, rapport au spectacle, modèle d'analyse intégratif, repères culturels 


\section{Introduction}

Les quatre disciplines du domaine des arts enseignées à l'école québécoise, soit l'art dramatique, les arts plastiques, la danse et la musique, sont de nature praxique, donc particulièrement «tournées vers l'agir et le faire » (Prairat, 1996, p. 15). D’ailleurs, les enseignantes et les enseignants spécialisés dans ce domaine sont souvent des praticiennes ou des praticiens, amateurs ou professionnels, de leur art. Ces personnes enseignantes, tout comme les spécialistes de l'éducation physique et à la santé, à titre d'exemple, peuvent continuer à pratiquer leur discipline en contexte de loisir, en milieu professionnel ou comme spectateur, au fil de leur carrière enseignante. Comme toutes les enseignantes et tous les enseignants spécialistes d'une discipline, les spécialistes des arts sont appelés à rester actifs dans leurs pratiques artistiques, qui nourrissent leur travail pédagogique. Ces pratiques, réalisées en parallèle ou en articulation à leur enseignement, leur permettent de s'alimenter en vue d'opérer une transposition didactique des savoirs artistiques dans leur classe et de continuer de se former en tant qu'artistes-pédagogues (Bonin, Duval, Blondin et Théberge, 2017). Nous émettons 1'hypothèse que, pour les enseignantes et les enseignants spécialistes en arts, leurs pratiques artistiques agissent de manière importante sur leur processus de transposition didactique, particulièrement quant à leur démarche personnelle d'appréciation de spectacles professionnels. Dans cette optique, nous nous intéresserons au spectacle professionnel comme objet de savoir et comme porte d'entrée dans la transposition didactique.

Le présent article a pour but d'interroger l'expérience d'appréciation de spectacles professionnels $^{1}$ que font les enseignantes et les enseignants spécialisés en arts, comme moyen pour réfléchir à la transposition didactique en enseignement des arts. Pour ce faire, nous exposerons d'abord le contexte spécifique de la transposition didactique en arts et les défis qu'il soulève. Ensuite, nous expliquerons comment les spectacles professionnels appréciés par les enseignantes et les enseignants sont conçus comme des objets de savoir et comme des repères culturels en didactique des arts. Enfin, nous présenterons comment deux modèles, soit la triple dimension de l'enseignement des arts (Raymond, 2014, 2018) et le rapport au spectacle (entre autres inspiré des travaux d'Émery-Bruneau, 2010, 2014), peuvent être croisés pour proposer un nouveau modèle d'analyse intégratif de la

${ }^{1}$ Bien que, dans cette étude, nous focalisions sur des disciplines liées aux arts vivants (théâtre, danse, arts du cirque), la réflexion se transpose aux œuvres visuelles et médiatiques ainsi qu'aux formes d'art hybrides. 
transposition didactique de l'expérience spectatrice de l'enseignante ou de l'enseignant en arts dans son enseignement.

\section{La transposition didactique en enseignement des arts}

Interroger les processus de transposition didactique dans l'enseignement des arts, c'est d'abord reconnaître la place que prennent les disciplines scolaires de nature praxique dans le curriculum prescrit, soient celles qui relèvent principalement d'une praxis (terme grec qui signifie l'action), notamment les arts et l'éducation physique et à la santé. Au Québec, ce n'est que depuis les années 1970 que les disciplines artistiques ont été progressivement intégrées au curriculum scolaire (Raymond et Lord, 2018), en commençant par les programmes d'arts plastiques et de musique et, dix ans plus tard, d'art dramatique et de danse (Gouvernement du Québec, 1981). Ces disciplines artistiques ont fini par trouver une place complémentaire, entre autres, aux côtés de celles que l'on qualifie de fondamentales, comme les mathématiques et la biologie appuyées sur des savoirs savants issus des sciences pures et naturelles (Chevallard, 1985/1991).

Les disciplines artistiques enseignées à l'école québécoise sont principalement fondées sur des pratiques de création, d'interprétation et d'appréciation d'œuvres, que Martinand (1986) a nommées les pratiques sociales de référence, c'est-à-dire celles qui ont cours dans la société et qui se manifestent dans des activités humaines et professionnelles. À ce chapitre, Raymond (2014) rappelle que dans les disciplines artistiques :

L'apprentissage des concepts «théoriques » [ou savoirs savants] passe d'abord par une expérimentation pratique proche de celle des artistes de profession. Les disciplines qui recourent à des pratiques autant qu'à des savoirs " savants » sont susceptibles de faire émerger de nouveaux savoirs qui n'existent pas dans la société, phénomène que Chervel (1988) a nommé les «créations didactiques ». Chevallard (1985) en a reconnu l'existence, mais a aussi critiqué cette forte tendance de l'école à se replier sur elle-même du point de vue de l'épistémologie. (p. 87)

$\mathrm{Si}$, depuis les années 2000, les études de transposition didactique sont relativement nombreuses dans le champ des didactiques des disciplines (Lenoir, 2020) ${ }^{2}$, notamment en

2 Selon Lenoir (2020), les didactiques des disciplines « [...] questionnent la nature même de la discipline enseignée, ses contours épistémologiques et le sens des contenus cognitifs à enseigner, et réfléchissent à leur aménagement, toujours du point de vue cognitif, pour les rendre accessibles aux élèves. Dit autrement, elles 
didactiques des langues (Gauvin et Boivin, 2012), des mathématiques (Musard, Robin, Nachon et Gréhaigne, 2008) et de l'éducation physique (Amans-Passaga, 2013; Terrisse, 2003), les études de transposition didactique en arts restent peu répandues, encore moins celles qui documentent les processus de transposition didactique interne du point de vue des savoirs enseignés par les enseignantes et les enseignants dans leur classe. Plus récemment, nous avons répertorié les études de Richard (2017) auprès de futures enseignantes et de futurs enseignants spécialisés en arts plastiques au Québec, d'ArnaudBestieu (2011) avec des spécialistes de l'éducation physique et sportive appelés à enseigner la danse en milieu scolaire français et Maizières (2012) sur l'enseignement de la musique à l'école primaire en France. Ce dernier insiste d'ailleurs sur la sélection des savoirs à enseigner en musique et, plus généralement en arts, sur les savoirs savants à privilégier et sur leur transposition en salle de classe.

\section{La place des œuvres professionnelles en enseignement des arts}

En didactique des arts, l'œuvre professionnelle, qu'elle soit théâtrale, chorégraphique, musicale ou visuelle, est au cœur des apprentissages et du développement des compétences disciplinaires chez l'élève (Gouvernement du Québec, 2001, 2003a et 2007). Au Québec, aucun corpus artistique n'est imposé, il revient aux enseignantes et aux enseignants de choisir des œuvres issus d'un répertoire à construire et d'utiliser des repères culturels pertinents, variés et significatifs. Bref, il appartient à chaque personne, nourrie par son expertise, sa vision de la discipline, sa connaissance des élèves qui lui sont confiés, sa planification et sa responsabilité pédagogiques, de les sélectionner et de les mettre au service des apprentissages. Dans ce processus, l'accès aux œuvres professionnelles doit apparaître au premier plan, comme l'indique d'ailleurs le descriptif de la troisième compétence des quatre disciplines artistiques, Apprécier des ouvres. Par exemple, pour la discipline danse :

Les extraits d'œuvres proviendront des périodes artistiques suivantes [...]. Ces extraits seront issus de différentes cultures, dont, si possible, celles des Premières Nations pour le deuxième cycle. Les élèves pourront aussi se référer aux spectacles de danse auxquels ils auront assisté. (Gouvernement du Québec, 2001, p. 235)

Depuis l'année scolaire 2018-2019, une nouvelle mesure gouvernementale vise à démocratiser les sorties culturelles pour tous les élèves du Québec en leur permettant de

étudient les phénomènes de mise en circulation des connaissances disciplinaires et elles théorisent les phénomènes d'enseignement de chaque discipline d'enseignement. » (p. 23) 
vivre de telles sorties gratuitement deux fois par année. Cette mesure issue de la politique culturelle provinciale Partout, la culture (Gouvernement du Québec, 2018) facilite la rencontre entre les groupes scolaires et les lieux culturels, et donc aussi avec les œuvres qu'ils présentent. Ce contexte devient maintenant des plus favorable pour intégrer les expositions et les spectacles professionnels aux contenus et aux savoirs prescrits dans les programmes, permettant ainsi aux élèves de vivre l'expérience de la rencontre avec l'art professionnel et aux enseignantes et aux enseignants de pouvoir réinvestir cette expérience esthétique dans leur enseignement des arts, qui vise en particulier le développement de la compétence d'appréciation des œuvres, mais aussi pour inspirer la création et l'interprétation. Nous nous intéresserons ici plus spécifiquement aux arts vivants, plus qu'aux œuvres visuelles et médiatiques, puisque ces spectacles sont au cœur du projetpilote Partenaires culturels CSSRS, qui représente notre terrain d'étude et que nous présenterons brièvement à la fin de cet article.

\section{Quelques concepts}

\section{Le spectacle comme objet de savoir scolaire}

Pour les enseignantes et les enseignants spécialistes d'une discipline artistique, les œuvres professionnelles, dont le spectacle fait partie, sont considérées comme des objets de savoir (Chevallard, 1985/1991) directement liés aux disciplines artistiques (art dramatique, arts plastiques, danse ou musique). Dans le Programme de formation de l'école québécoise (Gouvernement du Québec, 2001, 2003a, 2007), ce savoir essentiel (au primaire) ou contenu de formation (au secondaire) est catégorisé dans une rubrique appelée Répertoire pour l'appréciation d'ouvres dramatiques ou théâtrales, visuelles ou médiatiques, chorégraphiques et musicales.

\section{Le spectacle comme repère culturel}

Le spectacle professionnel peut aussi être considéré comme un savoir scolaire puisqu'il agit, selon son réinvestissement en classe, comme un repère culturel autant pour les enseignantes et les enseignants que pour les élèves. Les repères culturels sont définis comme des savoirs scolaires «[...] signifiants sur le plan culturel dont l'exploitation en classe permet d'enrichir le rapport à soi, aux autres et au monde » (Gouvernement du Québec, 2003b, p. 9). Ils sont inscrits dans les catégories de savoirs essentiels et de contenus de formation des programmes d'études, de façon plus ou moins explicite selon les disciplines. Par exemple, dans la discipline de la mathématique au primaire, les repères culturels sont explicitement présentés sous forme de liste, par cycle d'apprentissage 
(Gouvernement du Québec, 2001, p. 136). Une telle liste présente, par exemple, l'évolution dans l'écriture des nombres et les systèmes de numération comme repères culturels pour la mathématique. Toutefois, ce ne sont pas tous les domaines d'apprentissage qui proposent des repères culturels prédéterminés. Dans ce cas, il revient à la personne enseignante de les sélectionner et de les mobiliser dans des situations d'enseignement et d'apprentissage signifiantes pour les élèves. Un repère culturel est aussi « une ressource au service d'un savoir-faire, d'une compétence» (Simard, 2010, p. 97), ce qui permet d'inscrire les apprentissages dans un contexte et d'y donner un sens.

Dans les quatre disciplines artistiques au primaire, les repères culturels sont à peine évoqués. La même description en est faite pour chaque discipline. À titre d'exemple, voici ce qu'on peut lire dans le programme d'arts plastiques : "Tout au long de sa formation [...], l'élève est mis en contact avec de nombreux repères issus de sa culture immédiate ou se rapportant aux œuvres qu'il apprécie. Il est aussi appelé à faire des liens avec des repères culturels d'autres disciplines» (Gouvernement du Québec, 2001, p. 210). Dans les programmes d'études des quatre disciplines en arts au secondaire, la prescription est plus claire et détaillée. De grandes catégories de repères culturels sont identifiées, comme l'histoire de la discipline, la littérature, les lieux culturels, les carrières dans le domaine, des supports médiatiques, des œuvres du répertoire, etc., en laissant le soin aux personnes enseignantes de sélectionner les repères qui conviennent au contexte d'apprentissage et aux élèves visés. Nous présentons ici la description de la fonction du repère culturel pour la discipline art dramatique au premier cycle du secondaire :

Les repères culturels sont des éléments signifiants de la culture liés à l'art dramatique. Leur exploitation en classe permet à l'élève d'enrichir sa vision et sa compréhension du monde qui l'entoure. Ils lui permettent d'établir des liens concrets avec la discipline, d'en reconnaître les traces et les manifestations dans son environnement et de saisir le dynamisme et l'influence des arts dans la société. Leur choix doit s'effectuer en fonction de leur apport à la formation de l'élève, mais aussi en tenant compte des particularités régionales et de l'environnement immédiat de l'élève. » (Gouvernement du Québec, 2003a, p. 394)

Dans les programmes d'arts, le spectacle professionnel a bel et bien un statut d'objet de savoir scolaire, puisqu'il est un repère culturel à la fois signifiant et incontournable pour le domaine des arts. Il a été démontré (Nadeau, 2020) que, pour favoriser l'intégration de tels repères culturels signifiants au quotidien de la classe, il faut que les enseignantes et les enseignants soient convaincus de l'importance de la place à leur accorder. Le spectacle est 
particulièrement exploité dans les trois disciplines en arts de la scène (art dramatique, danse et musique), mais peut aussi être inspirant pour la création en arts plastiques.

\section{L'expérience esthétique du spectacle}

Être une spectatrice ou un spectateur est un rôle hautement actif où l'attention est sollicitée et où le sujet est interpelé autant par ses sens que par sa raison, ce qui correspond à une expérience dite esthétique. Pour définir ce concept, nous référons aux travaux de Schaeffer (2015) qui le décrit comme «une expérience attentionnelle exploitant les ressources cognitives et émotives communes, mais les infléchissant d'une manière caractéristique, inflexion en laquelle réside sa spécificité expérientielle » (p. 45). Ce n'est donc en aucun cas un rôle passif. Assister à un spectacle engage des émotions et une attention accrue, ce qui amène potentiellement la personne spectatrice à porter un regard sur elle-même et à mettre son imagination au travail (Allaire, 2020).

Si le spectacle, ce savoir de nature culturelle, participe du développement de la compétence à apprécier des œuvres artistiques chez l'élève, il contribue par extension à enrichir le rapport culturel que les enseignantes et les enseignants entretiennent eux-mêmes avec les savoirs à enseigner dans les programmes. Il convient donc de se demander comment l'expérience de spectateur des enseignantes et des enseignants spécialistes des arts, au primaire comme au secondaire, peut être réinvestie en classe. Comment ces spectacles, objets de savoirs scolaires et de références disciplinaires, peuvent-ils être transposés en classe du primaire ou du secondaire? Et quel modèle d'analyse intégratif permettrait de mieux saisir l'ensemble du processus de transposition didactique?

\section{Le spectacle professionnel transposé en classe d'arts}

Nous avons vu que l'objet-spectacle dans le Programme de formation trouve sa place dans le répertoire d'œuvres du domaine des arts et est exploité comme un repère culturel. Il s'agit donc d'un savoir scolaire à enseigner.

Comme le souligne Maizières, «c'est la question du savoir savant qui fait débat chaque fois qu'un auteur a souhaité interroger le concept de transposition didactique dans un autre champ disciplinaire [que les mathématiques] » (2012, p. 20). D'autres auteurs ont adapté les propositions de Chevallard (1985/1991) pour les actualiser dans leur domaine. C'est le cas de Martinand (1986), dans le champ des sciences et de la technologie, qui étend la notion de savoirs de référence aux pratiques sociales de référence, une notion qui 
s'applique particulièrement bien aux disciplines praxiques. Le concept de savoirs experts (Johsua, 1996, 1997) est aussi proposé pour démontrer que «toute discipline d'enseignement a des références extérieures, même si la référence n'est pas savante » (Mazière, 2012, p.20).

Nous présentons ici une démonstration de l'articulation possible de la chaîne de transposition didactique en enseignement des arts, considérant l'objet étudié et au regard de deux théories sur la transposition didactique. 
Tableau 1. Juxtaposition des chaînes de transposition didactique (TD) en enseignement des arts

\begin{tabular}{|c|c|c|}
\hline $\begin{array}{l}\text { La TD selon } \\
\text { Chevallard (1985) }\end{array}$ & $\begin{array}{l}\text { La TD selon Martinand } \\
\text { (1986) }\end{array}$ & $\begin{array}{l}\text { Contexte de la transposition didactique en } \\
\text { enseignement des arts à partir de l'objet- } \\
\text { spectacle professionnel apprécié par } \\
\text { l'enseignante ou l'enseignant en arts }\end{array}$ \\
\hline Savoirs savants & $\begin{array}{l}\text { Savoirs et pratiques sociales } \\
\text { de référence }\end{array}$ & $\begin{array}{l}\text { Le spectacle comme objet de savoir qui prend sa } \\
\text { source dans les pratiques professionnelles en arts } \\
\text { vivants }\end{array}$ \\
\hline \multicolumn{3}{|c|}{$\begin{array}{c}\text { Transposition didactique externe } \\
\text { Sélection et transformation par les agents de la noosphère }\end{array}$} \\
\hline Savoirs à enseigner & $\begin{array}{l}\text { Curriculum formel } \\
\text { (objectifs et programmes) }\end{array}$ & $\begin{array}{l}\text { Répertoire d'œuvres d'art dans les programmes } \\
\text { d'études en arts } \\
\text { (savoirs essentiels et contenus de formation } \\
\text { inscrits dans les programmes d'arts) }\end{array}$ \\
\hline \multicolumn{3}{|c|}{$\begin{array}{c}\text { Transposition didactique interne } \\
\text { Sélection et transformation par l'enseignante ou l'enseignant }\end{array}$} \\
\hline \multirow[t]{2}{*}{ Savoirs enseignés } & $\begin{array}{l}\text { Curriculum réel } \\
\text { (contenu de } \\
\text { l'enseignement) }\end{array}$ & $\begin{array}{l}\text { Conception ou actualisation de situations } \\
\text { d'apprentissage adressées aux élèves }\end{array}$ \\
\hline & $\begin{array}{l}\text { Appropriation par les élèves } \\
\text { (apprentissages affectifs et } \\
\text { durables) }\end{array}$ & $\begin{array}{l}\text { Partage de l'expérience d'appréciation du } \\
\text { spectacle par l'enseignante ou l'enseignant auprès } \\
\text { des élèves (sans qu'ils aient vu eux-mêmes le } \\
\text { spectacle) }\end{array}$ \\
\hline
\end{tabular}

Pour étudier les spécificités de la transposition didactique en classe d'arts, il aurait été possible de considérer différents savoirs scolaires, notamment ceux issus des savoirs essentiels (primaire) ou contenus de formation (secondaire). Nous privilégions le spectacle professionnel d'abord en raison de notre terrain d'étude, soit le projet-pilote Partenaires culturels CSSRS, mais aussi parce que les disciplines praxiques se nourrissent de la pratique professionnelle des artistes et que celle-ci évolue sans cesse. Pour enseigner les arts, on peut, bien sûr, s'appuyer sur des savoirs théoriques ou des manifestations historiques, mais pas uniquement. Comme passeurs culturels spécialistes en arts, il est de la responsabilité de chacune et de chacun de prendre part aux manifestations artistiques contemporaines (dans une posture d'acteur ou de spectateur) pour en témoigner dans son enseignement. Les traces de ces expériences s'incarnent par la transposition didactique. 


\section{Modèles théoriques et transposition didactique en arts à partir du spectacle}

Le contexte propre à la transposition didactique en enseignement des arts, soit le répertoire artistique peu défini, l'intégration des connaissances issues de la pratique artistique des personnes enseignantes et l'accès maintenant facilité aux spectacles, et l'importance du spectacle comme objet de savoir dans ce domaine d'enseignement nous amènent à nous intéresser à deux modèles qui peuvent éclairer les processus mis en œuvre par les spécialistes dans les classes d'arts. Nous nous intéressons à deux modèles complémentaires afin de marier le cognitif et le conatif, la raison et la passion, les compétences et les appétences, à la fois sur un plan individuel et sur un plan didactique. D'abord, la triple dimension de l'enseignement des arts articulée par Raymond $(2014,2018)$ pour illustrer les fondements de l'enseignement de ce domaine, en lien avec les trois compétences au programme. Ensuite, faisant un emprunt à la didactique des langues et appuyés sur les travaux d'Émery-Bruneau $(2010,2014,2018,2020)$ sur le rapport à la lecture littéraire, nous développons le concept de rapport au spectacle chez les enseignantes et les enseignants en arts. Finalement, nous proposons un nouveau modèle d'analyse intégratif à partir d'un croisement entre ces deux modèles pour décrire l'effet du rapport au spectacle des enseignantes et des enseignants en arts en relation avec les trois dimensions de l'enseignement des arts sur leurs pratiques didactiques.

\section{Triple dimension de la formation artistique à l'école}

Dans ses recherches qui ont permis de modéliser les pratiques de planification et d'enseignement de la danse dans une perspective de transposition didactique, ce que Chevallard (1985/1991) a défini comme la notion de transposition didactique interne, qui constitue le passage du savoir à enseigner vers le savoir enseigné, Raymond (2014) a identifié le processus de réflexion épistémologique qui a précédé la mise en forme des programmes d'enseignement des arts (Gouvernement du Québec, 2001, 2003a, 2007). Ce processus se traduit par la triple dimension de la formation artistique à l'école qui renvoie à l'éducation artistique, esthétique et culturelle constituant les fondements mêmes du domaine des arts. En prenant pour exemple la danse, comme art du spectacle, Raymond (2014) rappelle que :

Depuis l'Antiquité, l'artiste crée et interprète des œuvres alors que le spectateur apprécie le résultat de diverses formes d'expression artistiques. Ce registre d'expériences, que l'anthropologie associe à la catégorie de "la danse comme représentation [qui distingue les artistes des spectateurs, dont les rôles, normalement, ne sont pas interchangeables]" (Giurchescu, 2006), constitue le fondement épistémologique des trois compétences en arts de la scène (musique, danse et théâtre) 
et contribue à l'élaboration concertée des programmes d'études du domaine des arts. (p. 31)

Premièrement, l'éducation artistique sous-entend l'ensemble de la formation qui permet de placer l'élève dans une posture de créateur (pour toutes les disciplines) et d'interprète (en particulier dans les arts de la scène), favorisant ainsi le développement des compétences à créer et à interpréter des œuvres dramatiques, des images personnelles ou médiatiques, des danses ou encore des œuvres musicales. Si la formation artistique vise à éveiller la subjectivité, la sensibilité et la créativité des élèves, dans une perspective de transposition didactique, «elle exige aussi de l'enseignant [en arts] qu'il mette en jeu sa propre subjectivité dans la conception de situations d'enseignement-apprentissage » (Raymond, 2014, p. 34).

Deuxièmement, l'éducation esthétique concerne la formation reliée à l'appréciation d'œuvres d'art et d'objets culturels du patrimoine (peu importe la discipline artistique). Elle place l'élève dans la posture de spectateur, voire de critique d'art novice, ou amateur. Face aux objets d'art et de culture, l'élève est invité à porter sur le monde une attention esthétique (Ardouin, 1997) qui mobilise chez lui à la fois son regard et sa capacité de faire une lecture sensible de l'œuvre qui lui est présentée. Dans une perspective de transposition didactique, l'appréciation d'œuvres artistiques « pose la question du réinvestissement des expériences esthétiques vécues par les élèves dans leurs apprentissages scolaires et leur vie culturelle. » (Raymond, 2014, p. 37)

Enfin, l'éducation culturelle, qui se trouve complémentaire à l'éducation artistique (visant le développement des compétences pratiques à créer et à interpréter des œuvres) et à l'éducation esthétique (visant le développement de la compétence à apprécier des œuvres d'art et des objets culturels), constitue plus largement « l'ensemble de la formation grâce à laquelle l'élève découvre et partage son rapport avec le patrimoine [artistique], de tous les genres et de tous les temps, ainsi que son rapport avec les repères culturels issus d'autres champs de l'activité humaine ayant influencé l'évolution [des disciplines artistiques] » (Raymond, 2014, p. 38). Dans une perspective de transposition didactique, on définit la dimension culturelle à l'école comme des « actions favorisant l'exploitation de repères culturels signifiants associés à divers éléments constitutifs du Programme de formation (particulièrement, les savoirs essentiels propres à chacune des disciplines pour lesquelles un programme d'études est prescrit). » (Gouvernement du Québec, 2003b, p. 6). Ainsi, les enseignantes et les enseignants en arts, comme ceux des autres disciplines scolaires, sont appelés à les intégrer, tant dans la conception de leur enseignement que dans leur interaction éducative, en étant tout aussi ouverts aux repères culturels que les élèves 
peuvent, à leur tour, partager en classe. C'est de cette mise en synergie des repères culturels des personnes enseignantes et des élèves que le sens donné aux savoirs scolaires (Astolfi, 2008) peut progressivement se co-construire en classe.

Voici une représentation schématique de ces trois dimensions en relation avec les compétences du domaine des arts.

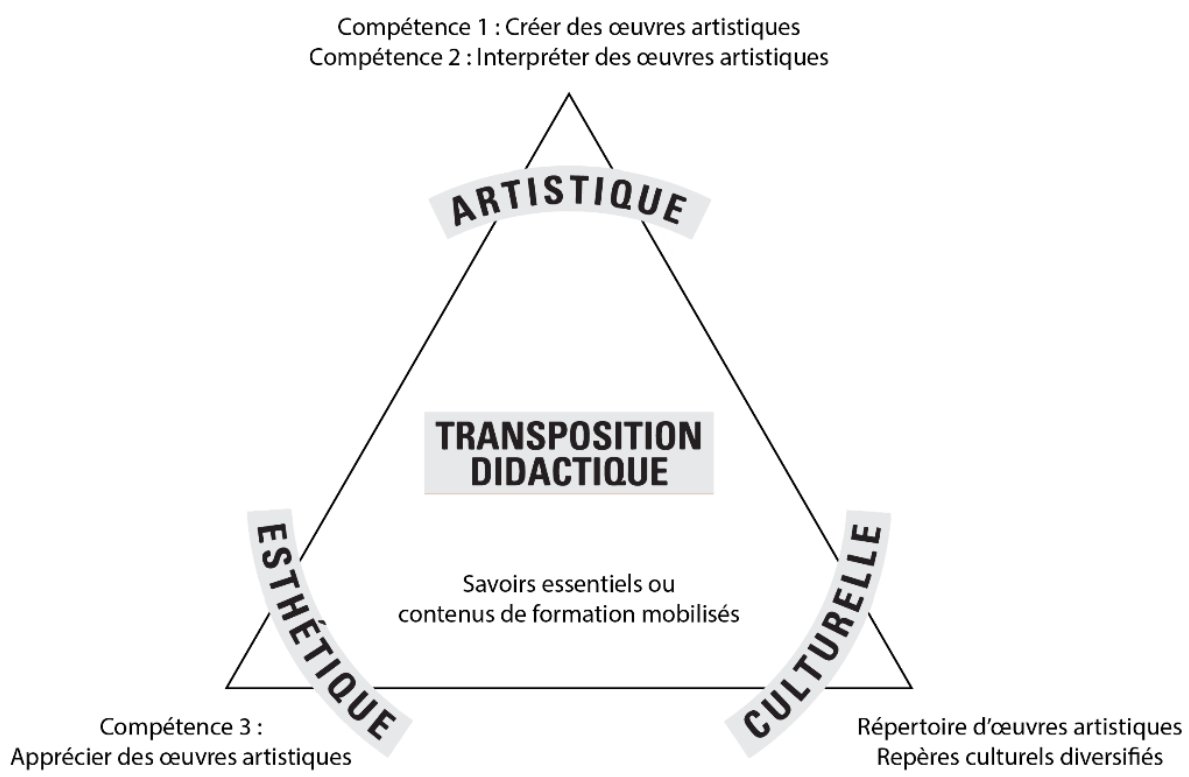

Figure 1. Triple dimension de la formation artistique à l'école selon Raymond $(2014,2018)$

\section{Rapport au spectacle en enseignement des arts}

Selon le Dictionnaire des concepts fondamentaux en didactique (Reuter, 2013), le rapport $\grave{a}$ en didactique « désigne la relation (cognitive mais aussi socio-affective) qu'entretient l'apprenant aux contenus et qui conditionne en partie l'apprentissage de ces derniers » (p. 185). Les études sur le rapport à se centrent sur la personne dans sa relation au savoir. Inspirés par l'importance de considérer le rapport aux savoirs des apprenantes et des apprenants afin de mieux ancrer leurs apprentissages, nous nous sommes particulièrement intéressés aux travaux d'Émery-Bruneau $(2010,2014,2018,2020)$ sur le rapport à la lecture littéraire, elle-même entre autres nourrie par les travaux du Groupe de Recherche Enseignement et Culture (Falardeau et Simard, 2007) sur le rapport à la culture. Les différents travaux sur le rapport à tiennent compte des représentations des participants sur l'objet étudié (dans notre cas, le spectacle) pour aborder la relation entretenue avec cet objet (Émery-Bruneau, 2010). 
Le travail d'Émery-Bruneau sur le sujet-lecteur et sa relation à la lecture littéraire, objet de savoir artistique, est constitué de deux plans : personnel et didactique (tenant compte du rapport à la lecture des élèves et de comment il va les former). La chercheuse a ainsi analysé le rapport à la lecture littéraire des étudiantes et des étudiants en enseignement du français dans toutes leurs dimensions et de les amener à en tenir compte pour le transformer et devenir des «sujets-lecteurs-enseignants engagés dans un rapport à la lecture littéraire pluridimensionnel, dynamique et conscient» (Ibid, p. i). Quatre dimensions interreliées composent les deux plans. Nous vous en présentons une synthèse dans le tableau 2.

Tableau 2. Dimensions du rapport à la lecture littéraire selon Émery-Bruneau (2010)

\begin{tabular}{ll}
\hline Dimension & Description \\
\hline Subjective & $\begin{array}{l}\text { Projets personnels, représentations, aspects psychoaffectifs, axiologiques et réflexifs } \\
\text { impliqués dans la lecture littéraire. }\end{array}$ \\
Sociale & $\begin{array}{l}\text { Rôle des autres, de l'école, des enseignants et rôle de la lecture littéraire dans le rapport } \\
\text { au monde du sujet. }\end{array}$ \\
Épistémique & $\begin{array}{l}\text { Nature, rôle et conceptions des savoirs et savoir-faire, ainsi que le rôle de la lecture } \\
\text { littéraire dans le développement des savoirs et savoir-faire. }\end{array}$ \\
Praxéologique & $\begin{array}{l}\text { Pratiques concrètes de lecture littéraire, soit les types de textes lus, les lieux et les } \\
\text { contextes de lecture, la situation et les objectifs de lecture, le temps et la fréquence de } \\
\text { lecture, ainsi que l'activité du sujet-lecteur pendant sa réception du texte. }\end{array}$ \\
\hline
\end{tabular}

Nous avons adapté son modèle pour pouvoir positionner chaque enseignante ou enseignant dans un rapport au spectacle comme individu et comme pédagogue, puis en constater les effets sur leurs choix didactiques et le type de processus de transposition mis en œuvre. À la lumière des travaux d'Émery-Bruneau, nous définissons le rapport au spectacle comme l'ensemble des relations dynamiques d'un spectateur avec un spectacle professionnel. Nous émettons l'hypothèse que plus le rapport au spectacle sur le plan personnel est complexe (articulé autour des quatre dimensions: subjective, sociale, épistémique et praxéologique), plus le rapport au spectacle sur le plan didactique le sera aussi. Ainsi, comme l'ont démontré les études de Falardeau et Simard (2017) sur le rapport à la culture, le spectacle sera plus susceptible d'être exploité comme un objet de savoir en classe, après être passé par le prisme de la transposition didactique.

\section{Proposition d'un modèle d'analyse intégratif}

Les deux modèles théoriques présentés plus haut sont articulés conjointement pour mieux saisir en quoi le rapport au spectacle de chaque enseignante ou enseignant en arts agit 
comme porte d'entrée vers la triple dimension de l'enseignement des arts et influence son processus de transposition didactique.

Sur le plan individuel, nous percevons la personne enseignante dans son rôle de spectatrice, dans sa relation avec les œuvres auxquelles elle assiste, sous les quatre dimensions étudiées : subjective, sociale, épistémique et praxéologique. À partir de la description d'une expérience marquante au spectacle, les références à chacune des dimensions sont notées pour identifier si l'une d'elles ressort comme dominante, ce qui nous informe de la nature $\mathrm{du}$ rapport au spectacle pour cette personne. Ensuite, nous considérons son rapport au spectacle, sur le plan didactique, dans les quatre mêmes dimensions dans son processus de transposition didactique. Par exemple, partage-t-elle aux élèves son amour pour les comédies musicales en témoignant de ses émotions vécues au théâtre (dimension subjective)? Place-t-elle les élèves en interaction lors de la création de spectacles (dimension sociale)? Enseigne-t-elle certaines notions du jeu d'acteur pour amener les élèves à mieux apprécier un spectacle (dimension épistémique)? Explore-t-elle une nouvelle façon d'approcher le jeu masqué en classe d'art dramatique après l'avoir observé dans un spectacle de danse contemporaine (dimension praxéologique)? La transposition didactique peut se déployer ainsi dans chacune des quatre dimensions du rapport au spectacle de la personne enseignante.

L'articulation des deux plans, personnel et didactique, est ensuite mise en relation avec la triple dimension de l'enseignement des arts à l'école. S'intéresser au rapport au spectacle des enseignantes et des enseignants en arts éclaire les trois pôles de ce modèle (dimensions artistique, esthétique et culturelle), dont voici une représentation schématique. 


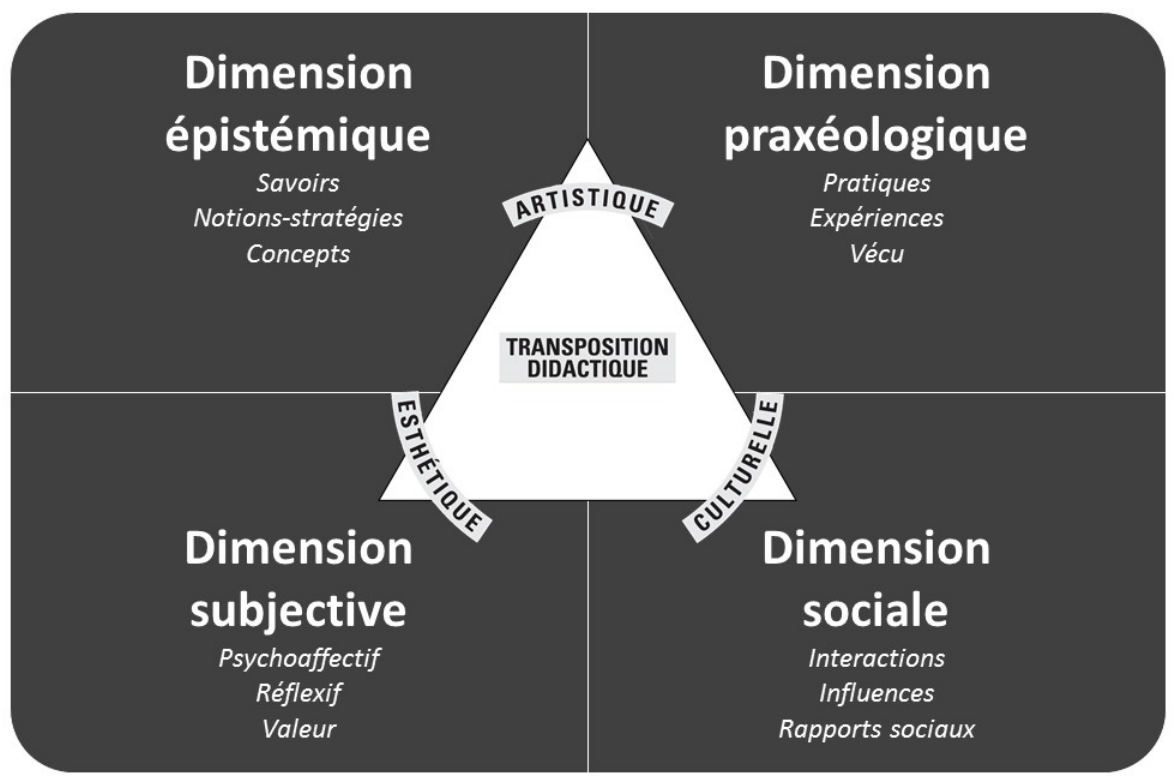

Figure 2. Relation entre les dimensions du rapport au spectacle de la personne-enseignante, la triple dimension de la formation artistique à l'école et la transposition didactique en arts (inspirée de : Raymond, 2014, 2018; Émery-Bruneau, 2010, 2014)

Rappelons d'abord que ces trois dimensions (artistique, esthétique et culturelle) guident les pratiques de transposition didactique en arts et que les enseignantes et les enseignants spécialistes de ce domaine sont souvent soumis à une tension entre leur bagage d'expériences personnelles de pratiques en arts et les savoirs scolaires des programmes d'études (Raymond, 2018), ce qui leur demande un effort «d'appropriation épistémologique des savoirs à faire apprendre dans une perspective d'éducation artistique, esthétique et culturelle » (p. 95).

Une fois l'expérience esthétique du spectacle vécue par l'enseignante ou l'enseignant d'arts analysée pour identifier son rapport au spectacle, un lien peut être établi avec sa façon de l'intégrer en classe. En effet, si le rapport au spectacle est surtout social pour une personne enseignante, comment cela influencera-t-il l'intégration de l'objet-spectacle en classe? Estce que la dimension culturelle sera la plus forte, nourrie par l'importance des interactions, de l'échange autour de l'expérience? Est-ce par cette fenêtre sur le spectacle comme expérience partagée que s'établira ensuite le développement de l'éducation esthétique et artistique, peut-être par la mise en œuvre d'un projet d'interprétation ou d'appréciation? Dans un autre cas, si le rapport au spectacle est surtout épistémique et que la personne 
enseignante est marquée par l'utilisation de l'espace dans une représentation en arts du cirque, est-ce qu'elle exploitera l'objet-spectacle qu'elle a vu d'abord par l'éducation artistique, en ciblant des éléments du programme qui permettent de traiter l'espace dans un exercice de création?

Cette proposition de modèle d'analyse est actuellement expérimentée dans un projet collaboratif en cours, notamment dans une étude de cas qui implique des enseignantes spécialistes des arts du primaire et du secondaire et dont voici un aperçu. Selon nous, le modèle d'analyse intégratif du rapport au spectacle et de sa transposition didactique en classe est porteur pour mieux saisir, dans toute sa complexité, les interrelations entre pratiques artistiques personnelles (dont celle de spectatrice ou de spectateur) et pratiques enseignantes.

\section{Survol méthodologique et pistes de réflexion}

\section{Le projet-pilote Partenaires culturels CSSRS}

Réalisé en partenariat par l'Université de Sherbrooke, le Centre culturel et le Centre de services scolaire de la Région-de-Sherbrooke (CSSRS) et financé par le ministère de la Culture et des Communications, le projet-pilote Partenaires culturels CSSRS propose à un millier de professionnelles et de professionnels de l'éducation un l'accès privilégié à des spectacles, des activités de médiation culturelle en arts et de la formation continue sur l'approche culturelle de l'enseignement dans le but de les amener à porter un regard critique sur leur rôle de passeurs, d'acteurs et de médiateurs culturels auprès des élèves.

Amener toutes les enseignantes et tous les enseignants à être en contact avec des œuvres professionnelles permettrait donc de nourrir leur développement professionnel. Tout particulièrement pour celles et ceux spécialisés en enseignement des arts, cela constitue une occasion de trouver de l'inspiration pour la conception didactique, au sens de planifier les situations d'enseignement et d'apprentissage (Gouvernement du Québec, 2020). Le projet-pilote Partenaires culturels CSSRS offre des finalités semblables à celles de l'éducation culturelle et esthétique mise en œuvre en classe d'arts au primaire et au secondaire, mais il s'adresse aux enseignantes et aux enseignants volontaires.

\section{Une brève méthodologie de la recherche en cours}

Une recherche longitudinale menée par une équipe de l'Université de Sherbrooke est en cours et analysera les retombées du projet-pilote sur le développement du rapport à la 
culture des enseignantes et des enseignants ainsi que ses effets auprès de leurs élèves. Un volet de la recherche se concentre sur les enseignantes et les enseignants en arts qui assistent de façon assidue aux spectacles proposés et qui ont pris part aux deux formations offertes. Dans cette recherche-intervention, réalisée sous la forme d'une étude de cas, le spectacle n'est pas imposé comme objet de savoir, mais les quatre enseignantes participantes considèrent que les spectacles qu'elles voient de façon personnelle laissent des traces dans leur enseignement. Elles se sont d'ailleurs volontairement engagées dans le projet pour réfléchir sur ces enjeux avec la chercheuse-intervenante. Dans ce contexte, l'expérience d'appréciation du spectacle représente un savoir issu de leur pratique professionnelle (expertes de leur discipline artistique et spectatrices expérimentées) qui est susceptible d'être réinvesti en classe et l'expérience d'appréciation que font les enseignantes (sans que les élèves aient apprécié eux-mêmes le spectacle) participent d'un processus de transposition didactique interne, ce que Raymond a nommé l'alternance entre les phénomènes de dialogue didactique intérieur et dialogue didactique interactif (Raymond, 2014; 2018; Raymond et Forget, 2020). Nous cherchons donc à comprendre comment ce processus de transposition didactique s'opère dans leur pratique enseignante en arts au regard du spectacle comme objet de savoir.

\section{Des résultats préliminaires}

Notre objectif est d'identifier et de décrire, à la lumière du modèle d'analyse intégratif proposé dans cet article, le rapport au spectacle de chaque participante pour déterminer comment ce rapport influence chaque enseignante en arts quand elle exploite un spectacle dans son enseignement. Le contexte pandémique et l'annulation des spectacles sur plusieurs mois a retardé la collecte de données prévue. Toutefois, au moment de rédiger ces lignes, la collecte est terminée et l'analyse thématique est en cours. Les premières analyses présentent déjà une corrélation entre les deux modèles. En effet, les portraits de chaque enseignante révèlent une cohérence entre la nature de leur pratique artistique, leur rapport au spectacle (dimension dominante) et le pôle le plus important de la triple dimension de la formation artistique dans leur pratique enseignante. Nous relevons aussi l'impact de la formation initiale et continue reçue et du parcours artistique personnel de la personne enseignante sur son processus de transposition didactique.

Par exemple, une enseignante spécialiste qui poursuit des projets de création personnels en plus de fréquenter les salles de spectacles assidument développe un rapport au spectacle particulièrement épistémique et privilégie, de façon inconsciente, le pôle artistique de la formation. Son rapport très personnel aux savoirs scolaires et ses savoirs disciplinaires lui 
permettent à la fois d'ancrer les apprentissages dans la pratique contemporaine, de les mettre en contexte dans l'histoire de la discipline et de s'engager dans le développement de compétences (ici, surtout des connaissances et des savoir-faire artistiques) des élèves par un accompagnement très personnalisé. Un autre cas, avec une enseignante formée dans une autre discipline que les arts et qui a volontairement changé de champ d'enseignement, convaincue de contribuer davantage à la formation générale des élèves en enseignant une discipline artistique, présente un rapport au spectacle principalement influencé par la dimension sociale, et son enseignement est clairement dirigé vers le pôle culturel de la formation artistique. Dans sa classe, l'élève est le point de départ des projets d'apprentissages et les savoirs scolaires issus du spectacle se greffent de façon informelle, surtout comme une occasion d'échanger sur des repères culturels et de partager des visions $\mathrm{du}$ monde. Bien entendu, la suite des travaux nuancera ces résultats partiels et nous permettra d'identifier et de décrire avec plus de précisons les traces du spectacle dans le processus de transposition didactique en enseignement des arts.

\section{Conclusion}

Le spectacle représente un objet de savoir scolaire pour les enseignantes et les enseignants en arts. Il trouve sa place dans le Programme de formation, dans la catégorie de savoir ou de contenu lié au répertoire d'œuvres. Puisque ce savoir à enseigner aux élèves permet le développement de la compétence à apprécier des œuvres, mais également celui des compétences praxiques, soit créer et interpréter, nous constatons l'importance de faciliter et d'encourager la fréquentation des spectacles et des œuvres professionnelles pour les enseignantes et les enseignants des quatre disciplines artistiques. L'expérience esthétique vécue par la personne enseignante peut devenir une source d'inspiration à la fois pour concevoir et faire vivre des situations d'apprentissage signifiantes aux élèves. Les premiers résultats nous permettent de constater que pour ces enseignantes et ces enseignants, les expériences esthétiques permettent de témoigner de l'évolution des pratiques artistiques à enseigner. Il s'agit aussi d'un moyen de modéliser auprès des élèves des attitudes d'ouverture et de curiosité face aux manifestations et œuvres artistiques qui sont nécessaires pour développer des compétences d'appréciation critique, en arts comme ailleurs. Il appert également que ces expériences nourrissent la créativité pédagogique des enseignantes et des enseignants en arts, et les amènent à se renouveler dans la conception de situations d'apprentissages. La suite de nos travaux nous permettra d'éprouver le modèle proposé dans cet article, puis d'en exposer les forces et les limites pour approfondir la description des processus de transposition didactique chez les enseignantes et les enseignants spécialistes issus de différentes disciplines artistiques. 


\section{Références}

Allaire, L. (2020). Le théâtre jeune public, les conditions favorables à l'expérience esthétique: trajectoires de deux créations contemporaines et interdisciplinaires destinées à l'extrémité des âges de l'enfance. [Mémoire de maîtrise, Université d'Ottawa]. http://dx.doi.org/10.20381/ruor-24787

Amans-Passaga, C. (2013). Analyse didactique de situations de partenariat professeur/intervenant, en ÉPS à l'école primaire. Recherches en didactiques, 2(16), 43-58. https://doi.org/10.3917/rdid.016.0043

Ardouin, I. (1997). L'éducation artistique à l'école. Paris : ESF éditeur.

Arnaud-Bestieu, A. (2011). L'incidence de l'épistémologie pratique des professeurs sur les savoirs co-construits en danse contemporaine: Analyse comparative de trois cas à l'école élémentaire. [Thèse de doctorat, Université de Toulouse II]. https://tel.archives-ouvertes.fr/tel-00634310/document

Astolfi, J.-P. (2008). La saveur des savoirs. Disciplines et plaisir d'apprendre. Issy-lesMoulineaux : ESF éditeur.

Bonin, H., Duval, H., Blondin, D. et Théberge, M. (2017). Identité(s) d'enseignants des arts: arts plastiques, danse, musique, art dramatique. Québec: Presses de l'Université Laval.

Chervel, A. (1988). L'histoire des disciplines scolaires. Réflexions sur un domaine de $\begin{array}{llll}\text { recherche. Histoire l'éducation, } & \text { (38), }\end{array}$ https://doi.org/10.3406/hedu.1988.1593

Chevallard, Y. (1985). La transposition didactique. Du savoir savant au savoir enseigné. Grenoble : La Pensée sauvage.

Chevallard, Y. et Johsua, M.-A. (1991). La transposition didactique. Du savoir savant au savoir enseigné (2e éd.). Grenoble : La Pensée sauvage.

Duchesne, C. et Leurebourg, R. (2012). La recherche-intervention en formation des adultes: une démarche favorisant l'apprentissage formateur. Recherches Qualitatives, 31(2), 3-24.

Émery-Bruneau, J. (2010). Le rapport à la lecture littéraire. Des pratiques et des conceptions de sujets-lecteurs en formation à l'enseignement du français à des intentions didactiques. [Thèse de doctorat, Université Laval]. https://doi.org/10.13140/RG.2.2.23179.69928

Émery-Bruneau, J. (2014). La littérature au secondaire québécois : conceptions d'enseignants et pratiques déclarées en classe de français. Lidil, 49, 71-89. https://doi.org/10.4000/lidil.3454 
Émery-Bruneau, J. (2018). Le rapport à la poésie d'enseignants du secondaire québécois : quelle progression entre les cycles/degrés? Tréma, 49, 29-42. https://doi.org/10.4000/trema.4526

Émery-Bruneau, J. (2020). Le noyau dur des pratiques d'enseignement de la poésie au secondaire québécois. $\quad$ Pratiques, 187-188, 1-23. https://doi.org/10.4000/pratiques.8907

Falardeau, É. et Simard, D. (2007). Le rapport à la culture des enseignants : proposition d'un cadre théorique. Nouveaux cahiers de la recherche en éducation,10(2), 131150. https://doi.org/10.7202/1018167ar

Gauvin, I et Boivin, M.-C. (2012). La théorie de la transposition didactique : un outil conceptuel pour décrire les savoirs grammaticaux élaborés en classe par les élèves. Bellaterra Journal of Teaching \& Learning Language \& Literature, 5(3), 10-24.

Gouvernement du Québec. (1981). Programme d'études en arts au primaire. Québec: ministère de l'Éducation.

Gouvernement du Québec. (2001). Programme de formation de l'école québécoise. Éducation préscolaire. Enseignement primaire. Québec : ministère de l'Éducation.

Gouvernement du Québec. (2003a). Programme de formation de l'école québécoise: Enseignement secondaire, premier cycle. Québec : ministère de l'Éducation.

Gouvernement du Québec. (2003b). L'intégration de la dimension culturelle à l'école: document de référence à l'intention du personnel enseignant. La culture toute une école! Québec : ministère de l'Éducation et ministère de la Culture et des Communications.

Gouvernement du Québec. (2007). Programme de formation de l'école québécoise: Enseignement secondaire, deuxième cycle. Québec : ministère de l'Éducation, du Loisir et du Sport.

Gouvernement du Québec. (2018). Partout la culture. Québec : ministère de la Culture et des Communications.

Gouvernement du Québec. (2020). Référentiel de compétences professionnelles. Profession enseignante. Québec : ministère de l'Éducation.

Johsua, S. (1996). Le concept de transposition didactique n'est-il propre qu'aux mathématiques? Dans C. Raisky et M. Caillot (dir.), Au-delà des didactiques, le didactique (64-73). Bruxelles : De Boeck.

Johsua, S. (1997). Le concept de transposition didactique peut-il étendre sa portée au-delà de la didactique des sciences et des mathématiques? Dans Roux, J.P. (Ed.), Skholê : Cahiers de la recherche et du développement. La didactique des sciences économiques et sociales $\left(\mathrm{n}^{\circ} 6\right)$. Montpellier : IUFM.

Kolb, D.A. (1984). Experiential learning. Englewood Cliffs (NJ) : Prentice-Hall. 
Lafortune, L. et Deaudelin C. (2001). Accompagnement socioconstructiviste. Montréal : Les presses de l'Université du Québec.

Lenoir, Y. (2020). Didactique : une approche sociohistorique du concept. Didactique, 1(1), 12-39. https://doi.org/10.37571/2020.0102

Maizières, F. (2012). Éducation musicale à l'école primaire : quels savoirs enseignés? Éduquer|Former, 43(1), 13-34.

Martinand, J.-L. (1986). Connaître et transformer la matière. Paris-Berne : Peter Lang.

Musard, D., Robin, J.-F., Nachon, M. et Gréhaigne, J.-F. (2008). La « composition sous influences » des curricula : un exemple en acrosport. Science \& motricité, (64), 6267. https://doi.org/10.3917/sm.064.0061

Nadeau, A. (2020). Conceptions d'enseignants du primaire sur leur rôle de passeur culturel : effets de dispositifs d'intégration de la dimension culturelle à l'école québécoise. [Thèse de doctorat, Université du Québec à Montréal]. Archipel. https://archipel.uqam.ca/13363/

Prairat, E. (1996). La discipline scolaire. Contenu, forme et visée. Se former plus : Pour apprendre et comprendre l'éducation et la formation, (62).

Paillé, P. et Mucchielli, A. (2016). L'analyse qualitative en sciences humaines et sociales. Paris : Armand Collin.

Perrenoud, P. (1996). Savoirs de référence, savoirs pratiques en formation des enseignants : une opposition discutable. Éducation et Recherche, (2), 234-250.

Raymond, C. (2014). Les pratiques effectives de la transposition didactique dans la planification et l'enseignement de la danse à l'école primaire québécoise : un mouvement dialogique intérieur et interactif. [Thèse de doctorat, Université de Sherbrooke]. Savoirs UdeS. https://savoirs.usherbrooke.ca/handle/11143/5483.

Raymond, C. (2018). La transposition didactique dans la planification et l'enseignement de la danse : faire appel aux dialogues intérieur et interactif. Dans H. Duval, C. Raymond et N. Turcotte (dir.), Faire danser à l'école (89-118). Québec : Presses de l’Université Laval.

Raymond, C. et Lord, M. (2018). L'intégration de la danse au curriculum scolaire québécois : portrait des programmes d'études des années 1980 et 2000. Dans H. Duval, C. Raymond et N. Turcotte (dir.), Faire danser à l'école (33-60). Québec : Presses de l'Université Laval.

Raymond, C. et Forget, M.-H. (2020). Pour un usage judicieux de l'analyse par théorisation ancrée dans le champ des didactiques. Éducation et Didactique, 14(1), 31-47. https://doi.org/10.4000/educationdidactique.5336

Reuter, Y. (2013). Dictionnaire des concepts fondamentaux des didactiques. Louvain-laNeuve : De Boeck Supérieur. 
Richard, M. (2015). Le projet Amalgame et son dispositif multimodal. Création et transposition de pratiques par de futurs enseignants en arts plastiques. Revue de recherches en littératie médiatique multimodale, 1. https://doi.org/10.7202/1047802ar

Schaeffer, J.-M. (2015). L'expérience esthétique. Paris : Gallimard.

Simard, D. (2010). La réforme de l'éducation au Québec : un trésor était caché dedans. Dans M. Mellouki (Ed.), Dans Promesses et ratés de la réforme de l'éducation au Québec (75-101). Québec : Les Presses de l'Université Laval.

Terrisse, A. (2003). Prise en compte du sujet et recherches en didactique des sports de combat : bilan et perspective. Science \& motricité, (50), 56-79. https://doi.org/10.3917/sm.050.0055

Zakhartchouk, J.-M. (1999). L'enseignant, un passeur culturel. Paris : ESF éditeur. 